\title{
Sensitivity analysis of mathematical models of signaling pathways
}

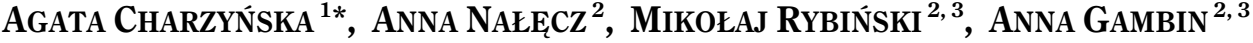 \\ ${ }^{1}$ Institute of Computer Science, Polish Academy of Sciences, Warsaw, Poland \\ ${ }^{2}$ Institute of Informatics, University of Warsaw, Warsaw, Poland \\ ${ }^{3}$ Mossakowski Medical Research Centre, Polish Academy of Sciences, Warsaw, Poland \\ *Corresponding author: A.Charzynska@phd.ipipan.waw.pl
}

\begin{abstract}
Modeling the dynamic behavior of signal transduction pathways is an important topic in systems biology. Mathematical models complement experimental technologies used to identify the molecular components and interactions in a system of interest. In this paper, we illustrate different types of mathematical approaches that are used to model signaling network behavior. Here, we review the basic methods of sensitivity analysis and apply them to the model of the system of membrane receptors. Four such receptors are considered: growth factor epidermal, low density lipoprotein, transferrin and vitellogenin receptor. We argue that application of sensitivity analysis methods provides an insight into how a signaling system controls the cell behavior.
\end{abstract}

Key words: membrane receptors, ordinary differential equations, stochastic modeling, chemical master equation, sensitivity analysis

\section{Introduction}

Mathematical modeling of complex biological systems can be carried out in a deterministic, stochastic or hybrid manner. The first one uses the classical differential equation theory, while the second one is based on the stochastic processes or stochastic differential equation theory. Both types of models are usually based on some simplifying assumptions that the temperature of a chemical environment is constant and, in a non-spatial setting, that the diffusion process occurs immediately, which ensures an even distribution of a substance over a limited volume.

Description of kinetics in most models stem from the classical chemical law of mass action. Deterministic models describe changes in mean concentrations of reagents over time, and they do not include the effect of fluctuations which occur in reality. This means that for given initial conditions, a deterministic model will always provide the same results.

Stochastic models describe the evolution of the probability distribution of all possible system states with respect to the time. These models are often expressed in the form of the Chemical Master Equation (CME), which is an alternative form of the Chapman-Kolmogorov equation. It describes the evolution of a distribution of a continuous-time Markov process over a set of all possible, discrete states (Kampen, 2007). A formal analysis of the CME is complex because of the large state space which covers all possible arrangements of molecules in the system, available through given reactions channels. Consequently, approximation methods were introduced, such as the Langevin equation (Gillespie, 2000) or the Fokker-Planck equation (cf. Sjöberg et al., 2009).

Many computational methods are available for both types of the mathematical model of reacting species. The basic method is numerical simulation, either of differential equations or the Markov process (so called stochastic simulations). These allow to approximate the reacting species evolution in time, thus, constituting a basis for further analysis of the model, such as the sensitivity analysis with respect to its parameters.

\subsection{Sensitivity analysis}

Sensitivity analysis (SA) investigates the relations between uncertain parameters of a model, and a property of the observable outcome (Saltelli et al., 2008), which represents some phenotypic features of the modeled system. SA has been used for various parameterization tasks of models of biological systems, such as finding essential parameters for research prioritization (Yue et al., 2008), identifying insignificant parameters 
for model reduction (Shankaran et al., 2006) or parameters clustering (Mahdavi et al., 2007).

Classically, sensitivity of the model is determined by the partial derivatives of the outcome with respect to its parameters. SA methods based on such quantities are called local as the derivative is taken at a fixed point in the state space of model parameters - at the parameter face value. Moreover, these methods belong to the class of one-factor-at-a-time (OAT) methods, because the net effect of a parameter on the property of the outcome is taken while assuming that all other factors are fixed. However, most of the biochemical reactions networks yield models of a nonlinear nature and "for these models, OAT methods can be of limited use if not outright misleading when the analysis aims to assess the relative importance of uncertain factors, and the model-free measures are needed, possibly independent of assumptions about the model structure (...)" (Saltelli et al., 2005). One solution is the investigation of the influence of simultaneous changes in parameters values by assessing higher order partial derivatives (Mahdavi et al., 2007), where the order depends on the nonlinearity level of the model. Nevertheless, it is still a local method, highly dependent on the given values of parameters. On the contrary, the so-called global sensitivity analysis (GSA) simultaneously examines a whole range of all input parameters values. Exemplary implementations of the GSA indices are the model-free, global sensitivity measures such as the variance decomposition (Saltelli et al., 2008), or the parameters space mapping method of Monte Carlo filtering (MCF) such as the multi-parameter sensitivity analysis (MPSA; Hornberger and Spear, 1981). In between, there are screening techniques which approximate the GSA indices. Screening techniques, such as the weighted average of local sensitivities (Bentele et al., 2004) or the elementary effects of Morris (1991), are global in the sense that they scan a whole range of parameters values, but they do that by using local OAT methods of sensitivity analysis for each analyzed set of parameter values. For a sake of clarity, if not explicitly stated otherwise, we will use a term local method meaning the local and OAT method, as well as a term global method meaning GSA method (Global and simulataneous).

Finally, there are SA methods tailored specifically to the stochastic models based on CME (Gunawan et al., 2005). These methods recognize that the response is in form of distribution rather than a single value correspon- ding, for instance, to the mean value. Consequently, for systems where a parameter disruption does not significantly influence the mean but significantly influences the distribution itself, the model-free SA indices can incorrectly indicate a lack of sensitivity of the model (cf. Degasperi and Gilmore, 2008).

\subsection{Organization of the paper}

In this paper, we briefly review the methods of mathematical modeling and SA applicable to models of signaling pathways. As an illustrative example, we compared both the deterministic and the stochastic model of a ligand-receptor binding and receptor internalization (Shankaran et al., 2007) by means of SA. To that end, we applied several methods: a local SA, a variance decomposition, and the Morris (1991) screening methods, including adjustment for the stochastic systems.

\section{Mathematical modeling of signaling pathways}

The biochemical model consists of $N$ kind of different substances (also called species) $\left\{S_{1}, \ldots, S_{N}\right\}$ and $M$ reactions between them. Each reaction may be presented in the following form:

$$
R_{m}: \underline{v}_{m 1} S_{1}+\underline{v}_{m N} S_{N} \stackrel{k_{m}}{\stackrel{v}{v}} \bar{v}_{m 1} S_{1}+\ldots+\bar{v}_{m N} S_{N},
$$

where $\underline{v}_{m n}$ and $\bar{v}_{m n}$ denote amounts of molecules of $n$-th substance that are accordingly substrate and product of $m$-th reaction and the coefficient $k_{m}$ denotes the reaction rate (speed) of $m$-th reaction.

\subsection{Deterministic modeling - ordinary differential equations derived from the mass action law}

The mass action law by Guldberg and Waage (dated at year 1864) constitutes the basis for chemical kinetics. According to this law, the probability of molecules collision at each time point depends only on the number of molecules in a volume unit of a modeled system and their mean kinetic energy, and the reaction rate is proportional to the concentration of all involved reagents. The Guldberg-Waage's law can be expressed using the following formula:

$$
\begin{aligned}
& \frac{\left[\dot{S}_{1}\right]}{v_{11}}=\ldots=\frac{\left[\dot{S}_{N}\right]}{v_{1 N}}=k_{1}\left[S_{1}\right]^{v_{11}} \cdot \ldots \cdot\left[S_{N}\right]^{\underline{v}_{1 N}} \\
& \frac{\left[\dot{S}_{1}\right]}{v_{M 1}}=\ldots=\frac{\left[\dot{S}_{N}\right]}{v_{M N}}=k_{M}\left[S_{1}\right]^{\underline{v}_{M 1}} \cdot \ldots \cdot\left[S_{N}\right]^{\underline{v}_{M N}},
\end{aligned}
$$


where $v_{m n}=\bar{v}_{m n}-\underline{v}_{m n}$ denotes a stoichiometric coefficient of $n$-th species in $m$-th reaction and [ $S_{n}$ ] denotes the concentration of $n$-th species. Finally $\dot{S}$ denotes a derivative of variable $S$ in respect to time. To simplify the notation and to avoid a division by zero without the restriction of the generality, it is assumed that all reactions consist only of species with a nonzero stoichiometric coefficient. In other words, all considered species take part in the reaction either as a substrate or as a product. By adding all above equations (2) we obtain the following differential equation describing the change in the concentration of a substance $S_{n}$ in the time flow:

$$
\frac{\mathrm{d}}{\mathrm{d} t}\left[S_{n}\right]=\sum_{m=1}^{M} v_{m n} k_{m}\left[S_{1}\right]^{\underline{\underline{v}}} \ldots\left[S_{N}\right]^{\underline{\underline{v}}_{m N}} .
$$

Commonly, the concentration is measured in a mole of the substance that coincides with one liter of a solution, i.e. the molar concentration ( $1 \mathrm{M}=1 \mathrm{~mol} / 1$ liter $)$. To express the amount of the substance in molecules number one has to multiply the molar concentration by $N_{A} V$ factor, i.e. $\left\langle \# S_{n}\right\rangle=N_{A} V\left[S_{n}\right]$ where $N_{A}$ denotes the Avogardo number and $V$ denotes the volume of the system. Accordingly, the differential equation (3) can be expressed as a change in the number of the molecules of an $n$-th substance in the time flow:

$$
\begin{aligned}
\frac{\mathrm{d}}{\mathrm{d} t}\left\langle \# S_{n}\right\rangle & =\sum_{m=1}^{M} \frac{v_{m n} k_{m}}{\left(N_{A} V\right)^{\underline{\underline{v}}}+\ldots+\underline{v}_{m N}-1} \\
& \times\left\langle \# S_{1}\right\rangle^{\underline{\underline{v}}_{m 1}} \ldots . .\left\langle \# S_{N}\right\rangle^{\underline{\underline{v}}_{m N}} .
\end{aligned}
$$

This operation is allowed only for systems with a large number of molecules, where one can assume that the change of the mean number of molecules is approximately continuous.

\subsubsection{Numerical integration}

A basis for the analysis of biochemical reactions networks expressed in the form of ODEs is their numerical integration. Among many methods for this task, the most popular are families of the predictor-corrector methods (e.g. Runge-Kutta) or the linear multistep methods (e.g. Adams-Moulton for non-stiff or backward differentiation formula for stiff systems; Butcher, 2003).

\subsection{The Chemical Master Equation (CME)}

CME is indeed an alternative form of Chapman-Kolomogorov equation for the Markov process (Kampen, 2007). A precise derivation of the $\mathrm{CME}$ was introduced by
Gillespie (1992). Assuming a thermal balance and an even distribution of the substance that is in a gaseous state, he proposed a mathematical formulation for the problem of one-directional bimolecular reactions. Moreover, he gave a reasoning for mono- and trimolecular reactions. Concerning that all two-directional reactions could as well be divided into two one-directional reactions and each reaction with more than two substrates can be expressed as a sequence of bimolecular reactions, the reasoning appears to be in-depth (Goutsias, 2007).

The following steps have been implied by Gillespie (1992) to derive the CME. The model is based on the same assumptions as in the Section 2.1 and it describes an evolution of a system with $M$ possible reactions involving $N$ different species.

The state of the system at each time point $t$ is described by the vector $\mathbf{X}(t)=\left(X_{1}(t), \ldots, X_{N}(t)\right)$, where $X_{n}(t)$ denotes the number of molecules of an $n$-th substance at time point $t$. Each of the $M$ reactions can be explicitly identified by three values:

- a vector of stoichiometric coefficients $v_{m}=\left(v_{m 1}, \ldots\right.$, $v_{m N}$ ) for species $S_{1}, \ldots, S_{n}$ respectively,

- the number of all possible substrates combination $h_{m}\left(x_{1}, \ldots, x_{N}\right)$ for each system's state $\mathbf{X}=\left(x_{1}, \ldots, x_{N}\right)$, $x_{n} \in \mathrm{N}$,

- the stochastic reaction's coefficient $c_{m}$ that is scalar such that $c_{m} \mathrm{~d} t$ denotes the probability of reacting of substrates combination randomly chosen from a substrates set of the reaction $R_{m}$ in the infinitesimal time interval $[t, t+\mathrm{d} t)$.

Assuming that the initial state of the system is known $\mathbf{X}\left(t^{0}\right)=\left(x_{1}^{0}, \ldots, x_{N}^{0}\right)=\mathbf{x}^{0}$ at the initial time point $t^{0}$, as well as coefficients $c_{m}$ of all stochastic reactions, the vector of stoichiometric coefficients $v_{m}$ and the function $h_{m}$ that indicates the number of all possible combination of substrates for each system state $\mathbf{X}$, one can determine the time evolution of the system. Let $\mathrm{P}\left(\mathbf{x}, t \mid \mathbf{x}^{0}, t^{0}\right)$ mean the conditional probability of the system being in state $\mathbf{x}=\left(x_{1}, \ldots, x_{N}\right)$ at a time point $t$, provided that $\mathbf{X}\left(t^{0}\right)=\mathbf{x}^{0}$. Under the assumptions of a uniform distribution of substances throughout the volume and thermal equilibrium, the probability of occurrence of a reaction in a short time interval depends only on the number of species at the time, and not on the previous states of the system. In disjoint time intervals, reactions occur independently. In an infinitesimal time interval $(t, t+\mathrm{d} t)$ the probability of: 
- occurrence of exactly one reaction $R_{m}$ is equal to $c_{m} h_{m}(\mathbf{x}) \mathrm{d} t+o(\mathrm{~d} t)$

- no reaction occurrence is $1-\sum_{m=1}^{M} c_{m} h_{m}(\mathbf{x}) \mathrm{d} t+o(\mathrm{~d} t)$,

- more than one reaction occurrence is $o(\mathrm{~d} t)$.

For each time point $t \in\left[t^{0}, t+\mathrm{d} t\right)$, there are three possible path evolutions:

- at time point $t$, the system is already in the final state $\mathbf{x}$ and no reaction has occurred in the time interval $[t, t+\mathrm{d} t)$,

- at time point $t$, the system was in one of $M$ previous states $\mathbf{x}-v_{m}$ and exactly one reaction $R_{m}$ had occurred in the time interval $[t, t+\mathrm{d} t)$,

- in the time interval $[t, t+\mathrm{d} t)$ occurred more than one reaction.

As these possible path evolutions are disjoint random events and there are no other possibilities, the probability of the system being in state $\mathbf{x}$ after time $t+\mathrm{d} t$ is equal to the sum of the probability of those three random events. Knowing that in the time intervals $\left[t^{0}, t\right)$ and $[t, t+\mathrm{d} t)$, reactions occur independently we obtain:

$$
\begin{aligned}
\mathrm{P}\left(\mathbf{x}, t+\mathrm{d} t \mid \mathbf{x}^{0}, t^{0}\right) & =\mathrm{P}\left(\mathbf{x}, t \mid \mathbf{x}^{0}, t^{0}\right) \\
& \times\left(1-\sum_{m=1}^{M} c_{m} h_{m}(\mathbf{x}) \mathrm{d} t+o(\mathrm{~d} t)\right) \\
& +\sum_{m=1}^{M} \mathrm{P}\left(\mathbf{x}-v_{m}, t \mid \mathbf{x}^{0}, t^{0}\right) \\
& \times\left[c_{m} h_{m}\left(\mathbf{x}-v_{m}\right) \mathrm{d} t+o(\mathrm{~d} t)\right] \\
& +o(\mathrm{~d} t) .
\end{aligned}
$$

Subtracting $\mathrm{P}\left(\mathbf{x}, t \mid \mathbf{x}^{0}, t^{0}\right)$, dividing by $\mathrm{d} t$ and going to limit with $\mathrm{d} t \rightarrow 0$, we obtain the Chemical Master Equation:

$$
\begin{aligned}
\frac{\partial}{\partial t} \mathrm{P}\left(\mathbf{x}, t \mid \mathbf{x}^{0}, t^{0}\right) & =\sum_{m=1}^{M} \\
& {\left[c_{m} h_{m}\left(\mathbf{x}-v_{m}\right) \mathrm{P}\left(\mathbf{x}-v_{m}, t \mid \mathbf{x}^{0}, t^{0}\right)\right.} \\
& \left.-c_{m} h_{m}(\mathbf{x}) \mathrm{P}\left(\mathbf{x}, t \mid \mathbf{x}^{0}, t^{0}\right)\right] .
\end{aligned}
$$

\subsection{Converting the deterministic into a stochastic model}

To obtain a stochastic counterpart of the deterministic model one has to determine the stochastic coefficients $c_{m}$ of each reaction $R_{m}$, and the function $h_{m}$ set for all possible system states. The stoichiometric coefficients vector $v_{m}$ is the same as in the deterministic model. The main difficulty is to determine stochastic coefficients $c_{m}$.

Although stochastic and deterministic models are based on the same assumptions, a stochastic model bet- ter reflects the described reality as it takes into account the impact of fluctuations on the mean value of the molecules number.

The following method for derivation of $c_{m}$ and $h_{m}$ is based on Wolkenhauer et al. (2004). As we remember, function $h_{m}$ returns the number of possible substrates combination for a reaction $R_{m}$ for each system state $\mathbf{x}=\left(x_{1}, \ldots, x_{N}\right)$. Knowing that an $m$-th reaction involves $\underline{v}_{m n}$ molecules of $n$-th kind, the number of possible combination is the following:

$h_{m}(\mathbf{x})= \begin{cases}\prod_{n=1}^{N}\left(\begin{array}{c}x_{n} \\ v_{m n}\end{array}\right) & \text { when } \forall n \in\{1, \ldots, N\} x_{n} \geq \underline{v}_{m n} \\ 0 & \text { otherwise. }\end{cases}$

For a sufficiently large number of $x_{n}$ the above formula can be approximated by:

$$
h_{m}(\mathbf{x})=\frac{\prod_{n=1}^{N} x_{n}^{\underline{v}_{m n}}}{\prod_{n=1}^{N}\left(\underline{v}_{m n} !\right)} .
$$

Let $\# R_{m}$ denotes the occurrence number of reaction $R_{m}$ in the time interval $[t, t+\mathrm{d} t)$. $\# R_{m}$ is a random variable with possible values in the set $\{0,1,2, \ldots\}$ and probability distribution as follows:

- probability of $\# R_{m}$ equal to 0 is $1-a_{m} \mathrm{~d} t-o(\mathrm{~d} t)$,

- probability of \# $R_{m}$ equal 1 to is $a_{m} \mathrm{~d} t$,

- probability of $\# R_{m}$ greater than 1 is negligible and denoted by $o(\mathrm{~d} t)$.

Where $a_{m}=h_{m} c_{m}$ denotes the propensity function of reaction $R_{m}$. The expected value of the occurrence number of a reaction $R_{m}$ in the time interval $[t, t+\mathrm{d} t)$ is

$$
\mathrm{E}\left(\# R_{m}\right)=\mathrm{E}\left(a_{m} \mathrm{~d} t\right)+o(\mathrm{~d} t) .
$$

The propensity function $a_{m}$, is not constant and depends on the system state $\mathbf{x}$. From the equation (4) one can obtain an alternative formula for the mean value of $R_{m}$ in the time interval $[t, t+\mathrm{d} t)$ :

$$
\mathrm{E}\left(\# R_{m}\right)=\frac{k_{m}}{\left(N_{A} V\right)^{\underline{\underline{v}_{m 1}}+\cdots+\underline{\underline{v}}_{m N}}-1} \prod_{n=1}^{N}\left(\mathrm{E}\left(\# S_{n}\right)\right)^{\underline{v}_{m n}} \mathrm{~d} t,
$$

where $\# S_{n}$ denotes the random variable for the number of molecules of $n$-th substance. By comparing both formulas (9) and (10) for the mean number of reactions $R_{m}$ in a very short time interval, one obtains the propensity function of reaction $R_{m}$ :

$$
\mathrm{E}\left(a_{m}\right)=\frac{k_{m}}{\left(N_{A} V\right)^{\underline{v}_{m 1}+\cdots+\underline{v}_{m N}}-1} \prod_{n=1}^{N}\left(\mathrm{E}\left(\# S_{n}\right)\right)^{\underline{v}_{m n}} .
$$


Using approximation (8) and definition of the propensity function $a_{m}$ :

$$
\mathrm{E}\left(a_{m}\right)=c_{m} \frac{\mathrm{E}\left(\prod_{n=1}^{N}\left(\# S_{n}\right)^{\underline{v}_{m n}}\right)}{\prod_{n=1}^{N}\left(\underline{v}_{m n} !\right)} .
$$

Finally, by the assumption of a lack of fluctuation and lack of correlation between molecules number of each substance, using relation $\mathrm{E}\left(\# S_{i}^{y_{m i}} \ldots \# S_{j}^{y_{m j}}\right)=\mathrm{E}\left(\# S_{i}\right)^{y_{m i}}$ ... $\mathrm{E}\left(\# S_{j}\right)^{y_{m j}}$, and comparing formulas (11) and (12), stochastic reaction's coefficients can be obtained:

$$
c_{m}=\frac{k_{m}}{\left(N_{A} V\right)^{\underline{v}_{m 1}+\cdots+\underline{v}_{m N}-1}} \prod_{n=1}^{N}\left(\underline{v}_{m n} !\right) \text {. }
$$

With this reasoning, one gets formulas (7) (or (8) for function $h_{m}$, as well as above formula (13) for reactions coefficients $c_{m}$. The last step of this stochastic model formulation is setting of the obtained values into the CME (6).

\section{Sensitivity analysis methods}

Sensitivity analysis is used to determine dependencies between input parameters and the results of the model. One can chose as input parameters for example initial concentrations of modeled species or reaction rates. Result of the model is most commonly defined as the density of species in any time point $t$. SA is very useful in mathematical modeling, as it describes dependencies between different elements of the model, it is also applicable to empirical experiments planning and enables verification of theoretical model results together with numerical and empirical results. SA also enables recognition of model's errors whether conceptual or in implementation. A more detailed description of different SA methods can be found in Campolongo et al. (2000).

SA techniques can be divided into three types: local methods, screening methods and global methods. In this section, all this three types are briefly characterized, some examples are additionally provided and subsequently applied to the ligand-receptor model.

\subsection{Local methods}

In empirical science, the most commonly used methods are local methods. Local analysis is set in the following manner. Let the model be dependent on $N$ para- meters $\left\{X_{1}, \ldots, x_{N}\right\}$ and let the result of the model be denoted by $y=f\left(x_{1}, \ldots, x_{N}\right)=f(\mathbf{x})$. It is assumed that the initial values of parameters were measured experimentally $\tilde{\mathbf{x}}=\left\{\tilde{X}_{1}, \ldots, \widetilde{X}_{N}\right\}$. The local SA describes the model fluctuation in the surrounding of $\tilde{\mathbf{x}}$ under specified parameters disruption.

The measure of influence of an $i$-th parameter is defined by partial derivative with respect to this parameter set in point $\tilde{\mathbf{x}}$.

$$
S_{i}=\frac{\partial}{\partial x_{i}} f(\tilde{\mathbf{x}}) .
$$

The value $S_{i}$ is called $i$-th sensitivity coefficient of the model. Local sensitivity coefficients are often normalized:

$$
S_{i}=\frac{\tilde{x}_{i}}{f(\tilde{\mathbf{x}})} \frac{\partial}{\partial x_{i}} f(\tilde{\mathbf{x}}) \text { and } S_{i}=\frac{\sigma\left(x_{i}\right)}{\sigma(f)} \frac{\partial}{\partial x_{i}} f(\tilde{\mathbf{x}}) .
$$

One normalization is with respect to the mean value and the other one is with respect to the standard deviation.

Normalization is important especially for models where the investigated parameters differ by several orders of magnitude. An alternative approach was described by Degasperi and Gilmore (2008), where to determine the sensitivity of the model to the parameter disruption, instead of absolute measure, the relative measure of the result change was used. The value:

$$
\begin{aligned}
& \frac{f\left(x_{1}, \ldots, x_{i-1}, x_{i}+p \% \cdot x_{i}, x_{i+1}, \ldots, x_{N}\right)}{p \% \cdot x_{i}} \\
& -\frac{f\left(x_{1}, \ldots, x_{i-1}, x_{i}, x_{i+1}, \ldots, x_{N}\right)}{p \% \cdot x_{i}}
\end{aligned}
$$

was substituted by

$$
\begin{aligned}
& f\left(x_{1}, \ldots, x_{i-1}, x_{i}+p \% \cdot x_{i}, x_{i+1}, \ldots, x_{N}\right) \\
- & f\left(x_{1}, \ldots, x_{i-1}, x_{i}, x_{i+1}, \ldots, x_{N}\right) .
\end{aligned}
$$

In this paper, both approaches using the normalized sensitivity coefficients and the relative sensitivity measure have been introduced for the ligand-receptor model.

An important advantage of the local SA is the simple concept and low computational cost. This approach is particularly useful in testing the models described with systems of complex differential equations with multiple variables and many parameters. The local methods are used in the first stage of model analysis which provides the overview of the problem. Local techniques have proven useful in inverse problems issues, in which estimation of the parameters describing the model is made on the basis of the observed results of the model (Campo- 
longo et al., 2000). More applications of local SA can be found in Turanyi (1990).

\subsection{Global methods}

The second and more profound approach is a GSA. Assuming that the value of each parameter $x_{i}$ varies in the range of values $\Omega_{i}$, one explores the whole variability space $\Omega=\Omega_{1} \times \cdots \times \Omega_{N}$ instead of limited surrounding of some point $\tilde{\mathbf{x}}=\left(x_{1}, \ldots, x_{N}\right)$ of parameters values. Nevertheless, finding a well-defined and computationally efficient measure of global sensitivity of the model is not easy.

To determine the single impact of $i$-th parameter on the outcome of the model, the following estimator is used:

$$
V_{i}=\mathrm{D}^{2}\left(\mathrm{E}\left(Y \mid X_{i}=x_{i}^{*}\right)\right),
$$

where $\mathrm{E}\left(Y \mid X_{i}=X_{i}^{*}\right)$ denotes the conditional expectation of variable $Y$, given that the value of $i$-th parameter is equal to $x_{i}^{*}$ (the $(\mathrm{N}-1)$-dimensional integral with respect to $(N-1)$ other parameters). While the variance takes into account all possible values of variable $X_{i}$ (onedimensional integral).

To measure the impact of the interaction between parameters $x_{i}$ and $x_{j}$ on the system outcome $y$, following measure is used:

$$
V_{i j}=\mathrm{D}^{2}\left(\mathrm{E}\left(Y \mid X_{i}, X_{j}\right)\right)-\mathrm{D}^{2}\left(\mathrm{E}\left(Y \mid X_{i}\right)\right)-\mathrm{D}^{2}\left(\mathrm{E}\left(Y \mid X_{j}\right)\right) .
$$

An analogous approach is used for larger groups of parameters. In this way, one obtains the distribution of the variance of variable $Y$ into components according to their increasing dimensionality:

$$
\mathrm{D}^{2}(Y)=\sum_{i} V_{i}+\sum_{i<j} V_{i j}+\ldots+V_{1 \ldots N} .
$$

The variance distribution (20) is unambiguous, only if the model parameters are independent (Sobol and Kucherenko, 2005). By dividing each of the components of this sum by the total variance of $V=\mathrm{D}^{2}(Y)$, global sensitivity coefficients of the model is obtained:

$$
S_{i_{1} \ldots i_{k}}=\frac{V_{i_{1} \ldots i_{k}}}{V} .
$$

Other possible approach is to use total sensitivity indices, introduced by Sobol (1993). This allows to estimate the impact of a single parameter on the variance of the model outcome, as well as the impact of all other possible combinations of parameters. For example, if a tested model depends on three parameters, the total sensitivity indices of $x_{1}$ is understood as: $S_{1}^{T}=S_{1}+S_{12}+$ $S_{13}+S_{123}$. In determining the indicator $S_{i}^{T}$, the following value is estimated:

$$
S_{i}^{T}=\frac{\mathrm{E}\left(\mathrm{D}^{2}\left(Y \mid X_{-i}\right)\right)}{\mathrm{D}^{2}(Y)},
$$

where, by $\mathrm{D}^{2}\left(Y \mid X_{-}\right)$the variance of variable $Y$ is denoted, for set parameters values except the value of $i$-th parameter.

$$
S_{1}^{T}=S_{1}+S_{12}+S_{13}+S_{123} .
$$

Sensitivity coefficients $S_{i}$ and $S_{i}^{T}$ have simple interpretation. The value of $S_{i}$ contains information on how much the total variance of the model could be reduced if the exact value of $i$-th parameter were known, while coefficient $S_{i}^{T}$ corresponds to the part of the total variance which would remain if the only unknown parameter were $X_{i}$ and the values of all other parameters were determined.

One of the most important methods of a global sensitivity analysis, allowing for the estimation of coefficients described above, is based on Fourier analysis of the Fourier Amplitude Sensitivity Test (FAST algorithm). Another important method is Sobol's method (Sobol, 1993), which consists of calculation of multidimensional integrals using the Monte Carlo method. The FAST method is described in detail below, and used to analyze the sensitivity of the ligand-receptor model. For other concepts of global SA, refer to Campolongo et al. (2000).

\subsubsection{The Fourier Amplitude Sensitivity Test (FAST)}

The classical FAST method enables to estimate only the first-order global sensitivity coefficients $S_{i}$ The extension of FAST method that enables to calculate the total sensitivity indices $S_{i}^{T}$, was presented in Saltelli et al. (1999).

Let $\mathbf{X}=\left(X_{1}, \ldots, X_{N}\right)$ be a random vector of parameters with a uniform distribution on the cube $\Omega=$ $[0,1]^{N}$. The model outcome $Y=f(\mathbf{X})$ is the random variable dependent on the parameters vector. Let $K$ be a curve along which one investigates the space of variability. And, let the curve $K$ be set by: 


$$
X_{i}(s)=G_{i}\left(\sin \left(\omega_{i} s\right)\right), \quad \forall i=1,2, \ldots, N,
$$

where $s$ takes all real values. Transformation $G_{i}$ is defined in such a way as to ensure the sampling of parameters in accordance with the adopted probability distribution. Let $\left\{\omega_{i}\right\}_{i=1}^{N}$ denote the set of different frequencies appropriately selected for each parameter. The choice of function $G_{i}$ was, in general, discussed in Cukier et al. (1978).

Let $f(s)=f\left(X_{1}(s), \ldots, X_{N}(s)\right)$ be the parameterization of the model outcome. Then the variance of the model outcome takes a form:

$$
\begin{aligned}
V & =\mathrm{D}^{2}(Y)=\lim _{T \rightarrow+\infty} \frac{1}{2 T} \int_{-T}^{T} f^{2}(s) \mathrm{d} s \\
& -\left[\lim _{T \rightarrow+\infty} \frac{1}{2 T} \int_{-T}^{T} f(s) \mathrm{d} s\right]^{2} .
\end{aligned}
$$

The model outcome $Y=f(s)$ as a periodic function can be presented in the form of a Fourier series:

$$
f(s)=\sum_{j=-\infty}^{+\infty}\left(A_{j} \cos (j s)+B_{j} \sin (j s)\right),
$$

where coefficients $A_{j}$ and $B_{j}$, are defined as follows:

$$
\begin{aligned}
& A_{j}=\frac{1}{2 \pi} \int_{-\pi}^{\pi} f(s) \cos (j s) \mathrm{d} s, \\
& B_{j}=\frac{1}{2 \pi} \int_{-\pi}^{\pi} f(s) \sin (j s) \mathrm{d} s .
\end{aligned}
$$

Adopting the reasoning from Saltelli et al. (1999), let $\Lambda_{j}=A_{j}^{2}+B_{j}^{2}$ denote the amplitude spectrum of the function $f(s)$. As per the Parseval theorem it is:

$$
\sum_{j \in Z} \Lambda_{j}=\frac{1}{2 \pi} \int_{-\pi}^{\pi} f^{2}(s) \mathrm{d} s .
$$

By comparing the equations (25) and (28) and using the relationship between Fourier coefficients $A_{-j}=A_{j}$, $B_{-j}=-B_{j}, \Lambda_{-j}=\Lambda_{j}$ the variance estimator for random variable $Y$ is obtained:

$$
\hat{V}=\sum_{j \in \mathrm{Z}-\{0\}} \Lambda_{j}=2 \sum_{j=1}^{+\infty} \Lambda_{j} .
$$

At the same time, we obtain the estimator for the conditional expectation of variable $Y$ given $X_{i}$ (comp. (18))

$$
\hat{V}_{i}=\sum_{p \in Z-\{0\}} \Lambda_{p \omega_{i}}=2 \sum_{p=1}^{+\infty} \Lambda_{p \omega_{i}} .
$$

Finally, having estimators values $\hat{V}$ and $\hat{V}_{i}$ one can estimate the value of sensitivity coefficients for $i$-th parameter: $\hat{S}_{i}=\hat{V}_{i} / \hat{V}$.

\subsection{Screening methods}

Screening methods are a variety of qualitative global sensitivity analysis. They allow to explore the entire variability space of parameters $\Omega$ at a relatively low computational cost. The purpose of the analysis is to determine which parameters significantly influence the outcome of the model, and to identify those parameters that affect the outcome negligibly. Screening methods allow to rank the parameters in order of a decreasing effect on the model outcome, but not the quantitative relationship of this impact. Screening methods are applied to determine the sensitivity of complex models, depending on many factors, as well as models with a high evaluation cost. One of the screening methods is the Morris method, presented below, used for SA of the ligand-receptor model.

\subsubsection{The Morris method}

The Morris method (Morris 1991) can be classified as a technique on the borderline of global and local methods, because it takes into account the whole variability space $\Omega$, however, at each test point of $\Omega$, one carries a local SA. The algorithm of the Morris method belongs to screening methods. It determines which parameters have a (i) linear and additive (ii) resulting from the nonlinear interaction of the other parameters, or (iii) negligible influence on the model outcome.

Without any loss of generality, one can assume that the variability space is an $N$-dimensional unit cube $\Omega$ $=[0,1]^{N}$. Let us consider the discrete variability space in which each parameter can assume $p$ values:

$$
x_{i} \in\left\{0, \frac{1}{p-1}, \frac{2}{p-1}, \ldots, 1\right\},
$$

one receives an $N$-dimensional $p$-leveled network of parameters covering the space $\Omega$. Let $\Delta$ denote a fixed multiple of the fraction $1 / p-1$. Let $\mathbf{x}=\left(x_{1}, \ldots, x_{N}\right)$ be a chosen point of the network, so that the point $\left(x_{1}, \ldots\right.$, $\left.x_{i-1}, x_{\mathrm{i}}+\Delta, x_{i+1}, \ldots, x_{N}\right)$ also belongs to the network. The elementary effect of $i$-th parameter disturbance at point $\mathbf{x}$ can be determined by the following difference quotient:

$$
d_{i}(\mathbf{x})=\frac{f\left(x_{1}, \ldots, x_{i-1}, x_{i}+\Delta, x_{i+1}, \ldots, x_{N}\right)-f(\mathbf{x})}{\Delta} .
$$

This is an approximation for a partial derivative of the model outcome in respect to parameter $x_{i}$ counted in point $\mathbf{x}$ at the network. The method provides for each 
parameter $x_{i}$ an independent drawing of $R$ points at the network corresponding to different sets of parameters of the model, and calculation of the difference quotients $d_{i}$ for the drawn parameters, creating sample $F_{i}$

Based on the characteristics of sample $F_{i}$, one can infer regarding the impact of a given parameter on the outcome of the model. A feature that allows to rank the parameters according to their decreasing significance is a measure of location, for example the mean value. A large absolute value of the mean of the sample $\mathrm{E}\left(F_{i}\right)$ indicates that parameter $x_{i}$ is important to the model outcome and it cannot be ignored in a subsequent analysis. On the other hand, a small absolute mean value indicates a negligible effect of the $i$ th parameter on the outcome of the model. Therefore, to simplify further analysis, this parameter can be treated as a constant.

Dispersion of the sample $F_{l}$ measured for example with the standard deviation (Morris, 1991), provides information on the nature of the impact on the outcome of a given parameter. A large dispersion of the sample means a nonlinear impact of parameter $x_{i}$ on the model outcome, or influence of this parameter on the model outcome by interacting with other parameters of the model.

\subsection{Stochastic sensitivity analysis}

The so-far described methods of SA have been developed for deterministic ODE models. The stochastic sensitivity analysis requires a slightly different approach as presented by Degasperi and Gilmore (2008) and Gunawan et al. (2005).

In the case of deterministic models in an SA for a given set of input parameters always the same results are obtained. Contrary to this, the result of a stochastic simulation model is a random variable and for fixed initial data it takes, different value each time. The distribution of this random variable can be estimated from many simulations.

A comparison of the results of a deterministic model calculated for different sets of input parameters does not cause any difficulties. However, in the case of stochastic models there is a need to measure the distance between two probability distributions. One possibility is to compare the average of both distributions. Doing so, however, forces the assumption of normality of these distributions and does not take into account the variance of the results. Therefore, a better solution is to use measures of distance between distributions, for example, the distance of histograms or the Kolmogorov distance.

Let $S$ denote a sample of size $|S|$ drawn from some probability distribution, and let $T$ denote a sample drawn from another distribution, with $|T|$ elements. A distance of histograms for these samples is defined as follows (Degasperi and Gilmore, 2008):

$$
D_{\text {hist }}(S, T)=\sum_{i=1}^{k}\left|\frac{\sum_{j=1}^{|S|} \chi\left(s, I_{i}\right)}{|S|}-\frac{\sum_{j=1}^{|T|} \chi\left(t, I_{i}\right)}{|T|}\right|,
$$

where $k$ denotes the column number of the histograms, $I_{i}$ denotes the range of values of $i$-th histograms' column, $s$ and $t$ are elements of the sets $S$ and $T$ accordingly, and $\chi\left(\cdot, I_{i}\right)$ denotes the characteristic function of an $i$-th interval $I_{i}$

Notice that a self distance of histograms (two samples from the same distribution) will be generally different from zero. Therefore, in the assessment of the distance of histograms for the results obtained for the initial data and the perturbed data, one should compare this distance with the distance of histograms calculated for the two sets drawn from the initial distribution. If both values are comparable, then one cannot conclude a significant influence of parameters disturbance on the outcome of the model.

An alternative measure for a distance of distributions is the Kolmogorov distance, which is defined as the maximum of absolute value of distance of empirical distribution functions at each point $t$ :

$$
D_{K}(S, T)=\max _{t}\left|F_{S}(t)-F_{T}(t)\right| .
$$

Also for this measure, the distance between two samples from the same distribution is generally different from zero.

\section{Case-study of a ligand-induced receptor activa- tion and receptor internalization}

As an illustrative example of the presented ideas we consider the mathematical model of ligand-induced receptor system from Shankaran et al. (2007). We transformed the classical deterministic version into a stochastic model. For both models, appropriate SAwere applied.

The model reflects a system of cell surface receptors in a single cell and describes the time evolution of three different species: ligands $L$ in the intercellular space, 
free receptors $R$ on a cell membrane and ligand-receptor complexes $C$. One of the model's parameters is the volume measure of the intercellular space that falls for a single cell. All processes that can occur in the modeled system are shown in Figure 1. Ligands are emitted into the intercellular space with intensity $f$ from some source $S$. Ligands bind with free receptors on a cell membrane into complexes $C$ with a factor $k_{\text {on }}$. The complexes can again fall into parts with a factor $k_{\text {off }}$ or be internalized into a cell with a factor $k_{\mathrm{e}}$. The cell produces new receptors with an intensity $q$ and absorbs free receptors (nonbound with ligands) with an intensity $k_{\mathrm{t}}$. In summary, the system of reactions is the following:

$$
\begin{array}{ll}
\stackrel{f}{0 \rightarrow L} & C \rightarrow k_{\text {off }} \\
\underset{0}{q \rightarrow R} & \stackrel{k_{\mathrm{e}}}{\rightarrow} L+R \\
\stackrel{k_{\text {on }}}{\rightarrow} & \stackrel{k_{\mathrm{t}}}{k_{\mathrm{t}}} \\
L+R & \quad R \rightarrow 0 .
\end{array}
$$

Using the Mass Action Law, two differential equations systems can be derived, one for the distribution of reagents:

$$
\begin{aligned}
& \frac{\mathrm{d}}{\mathrm{d} t}[L]=f-k_{\mathrm{on}}[L][R]+k_{\mathrm{off}}[C] \\
& \frac{\mathrm{d}}{\mathrm{d} t}[R]=q-k_{\mathrm{on}}[L][R]+k_{\mathrm{off}}[C]-k_{\mathrm{t}}[R] \\
& \frac{\mathrm{d}}{\mathrm{d} t}[C]=k_{\mathrm{on}}[L][R]-k_{\mathrm{off}}[C]-k_{\mathrm{e}}[C]
\end{aligned}
$$

and the other for the mean number of molecules:

$$
\begin{aligned}
& \frac{\mathrm{d}}{\mathrm{d} t}\langle \# L\rangle=f N_{A} V-\frac{k_{\mathrm{on}}}{N_{A} V}\langle \# L\rangle\langle \# R\rangle+k_{\mathrm{off}}\langle \# C\rangle \\
& \frac{\mathrm{d}}{\mathrm{d} t}\langle \# R\rangle=q N_{A} V-\frac{k_{\mathrm{on}}}{N_{A} V}\langle \# L\rangle\langle \# R\rangle+k_{\mathrm{off}}\langle \# C\rangle-k_{\mathrm{t}}\langle \# R\rangle \\
& \frac{\mathrm{d}}{\mathrm{d} t}\langle \# C\rangle=\frac{k_{\mathrm{on}}}{N_{A} V}\langle \# L\rangle\langle \# R\rangle-k_{\mathrm{off}}\langle \# C\rangle-k_{\mathrm{e}}\langle \# C\rangle .
\end{aligned}
$$

Following Shankaran et al. (2007) we investigate four types of receptors:

- epidermal growth factor receptor, (EGFR), which stimulates cell division and plays an important role in the process of tumor formation,

- transferrin receptor (TfR), responsible for the transport of iron into cells,

- low-density lipoprotein receptor (LDLR), transporting cholesterol into cells,
- vitellogenin receptor (VtgR), which mediates the uptake of vitellogenin (Vtg) in oocyte development.

Table 1 contains the experimentally measured reaction rates for these four types of receptors.

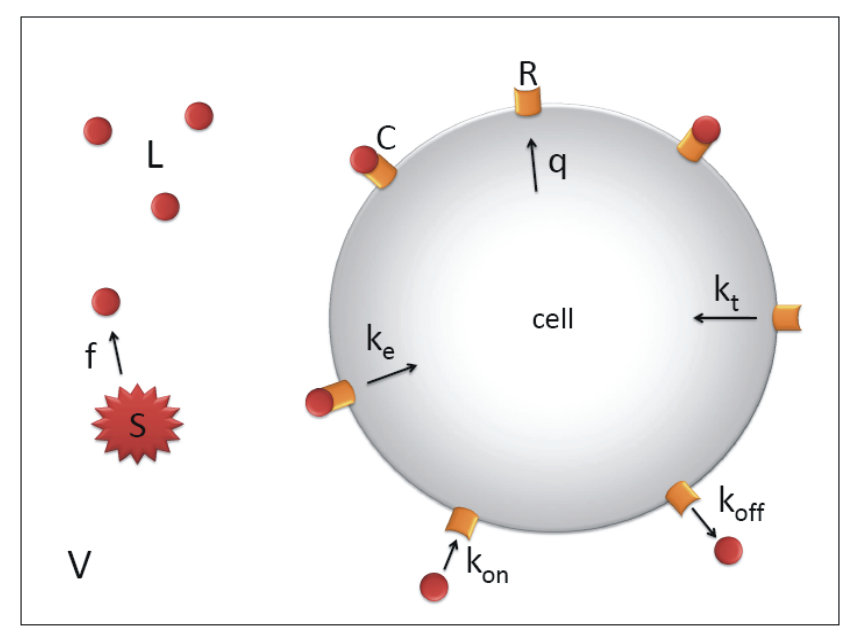

Fig. 1. Reactions scheme in the ligand-induced receptor system, see Shankaran et al. (2007). $V$ - volume measure for the cell, $L$ - ligands, $S$ - source of ligands emission, $R$ - free receptors, $C$-complexes of receptors bound with ligands

If there is no ligands emission into the intercellular space and therefore no complexes of ligands and receptors can be formed, then the number of free receptors on the cell surface is described simply by:

$$
\frac{\mathrm{d}}{\mathrm{d} t}\langle \# R\rangle=q N_{A} V-k_{\mathrm{t}}\langle \# R\rangle .
$$

In this case, the stationary state of the mean number of receptors is $R_{\mathrm{T}}=q N_{A} V / k_{\mathrm{t}}$.

This implies that the reaction rate of free receptors production is $q=R_{\mathrm{T}} k_{\mathrm{t}} / N_{A} V$.

The reaction rate $f$ describing a ligand emission rate is one of the model parameters, so as the intercellular volume for cell $V$. Typically $V$ is between $4 \times 10^{-13} 1$ (cell volume) and $4 \times 10^{-10} 1$ (for fluid tissue). The value used in the computation of the model is $V=4 \times 10^{-10} 1$.

\subsection{Stochastic version of the model}

Let $\mathbf{X}(t)=\left(x_{L}, x_{R}, x_{C}\right)$ denote a random vector of a modeled system state in time point $t$, where $x_{L}$ denotes the number of ligands in the intercellular space, $x_{R}$ denotes the number of free receptors and $x_{C}$ denotes the number of the ligand-receptor complexes. Basing on the modeled reactions scheme (35) one can determine the stoichiometric coefficients: 
Table 1. Values of reaction rates for receptors EGFR, LDLR, TfR and VtgR

\begin{tabular}{c|c|c|c|c|c}
\hline Receptor & $\begin{array}{c}k_{\text {on }} \\
M^{-1} \mathrm{~min}^{-1}\end{array}$ & $\begin{array}{c}k_{\text {off }} \\
\min ^{-1}\end{array}$ & $\begin{array}{c}k_{\mathrm{e}} \\
\min ^{-1}\end{array}$ & $\begin{array}{c}k_{\mathrm{t}} \\
\min ^{-1}\end{array}$ & $\begin{array}{c}R_{\mathrm{T}} \\
\text { no of molecules }\end{array}$ \\
\hline EGFR & $9.70 \times 10^{7}$ & 0.2400 & 0.150 & 0.020 & $2.0 \times 10^{5}$ \\
\hline LDLR & $2.48 \times 10^{6}$ & 0.0354 & 0.195 & 0.195 & $2.0 \times 10^{4}$ \\
\hline TfR & $3.02 \times 10^{6}$ & 0.0900 & 0.600 & 0.600 & $2.6 \times 10^{4}$ \\
\hline VtgR & $5.76 \times 10^{4}$ & 0.0700 & 0.108 & 0.108 & $2.0 \times 10^{11}$ \\
\hline
\end{tabular}

$$
\begin{array}{ll}
v_{1}=(1,0,0) & v_{4}=(1,1,-1) \\
v_{2}=(0,1,0) & v_{5}=(0,0,-1) \\
v_{3}=(-1,-1,1) & v_{6}=(0,-1,0) .
\end{array}
$$

Moreover using formulas (7) and (13), one can obtain the propensity function of each modeled reaction:

$$
\begin{array}{ll}
a_{1}\left(x_{L}, x_{R}, x_{C}\right)=N_{A} V f & a_{4}\left(x_{L}, x_{R}, x_{C}\right)=k_{\text {off }} x_{C} \\
a_{2}\left(x_{L}, x_{R}, x_{C}\right)=N_{A} V q & a_{5}\left(x_{L}, x_{R}, x_{C}\right)=k_{\mathrm{e}} x_{C} \\
a_{3}\left(x_{L}, x_{R}, x_{C}\right)=\frac{k_{\mathrm{on}}}{N_{A} V} x_{L}, x_{R} & a_{6}\left(x_{L}, x_{R}, x_{C}\right)=k_{\mathrm{t}} x_{R} .
\end{array}
$$

Once the stoichiometric vectors $v_{m}$ and the propensity functions $a_{m}(\mathbf{x})$ are known, it is possible to formulate the Chemical Master Equation corresponding to the stochastic model of the system describing the ligand-receptor evolution. In order to simplify the notation, we denote the probability $\mathrm{P}\left(\mathbf{x}, t \mid \mathbf{x}^{0}, t^{0}\right)$ of the system being in state $\mathbf{x}=\left(x_{L}, x_{R}, x_{C}\right)$ in time point $t$ given the initial state of the system $\mathbf{X}\left(t^{0}\right)=\mathbf{x}^{0}$, by $p_{t}(\mathbf{x})$. For this model, the CME has the following form:

$$
\begin{aligned}
\frac{\partial}{\partial t} p_{t}\left(x_{L}, x_{R}, x_{C}\right) & =N_{A} V f p_{t}\left(x_{L}-1, x_{R}, x_{C}\right) \\
& -N_{A} V f p_{t}\left(x_{L}, x_{R}, x_{C}\right) \\
& +N_{A} V q p_{t}\left(x_{L}, x_{R}-1, x_{C}\right) \\
& -N_{A} V q p_{t}\left(x_{L}, x_{R}, x_{C}\right) \\
& +\frac{k_{\mathrm{on}}}{N_{A} V}\left(x_{L}+1\right)\left(x_{R}+1\right) p_{t}\left(x_{L}+1, x_{R}+1, x_{C}\right) \\
& +\frac{k_{\mathrm{on}}}{N_{A} V} x_{L} x_{R} p_{t}\left(x_{L}, x_{R}, x_{C}\right) \\
& +k_{\mathrm{off}}\left(x_{C}+1\right) p_{t}\left(x_{L}, x_{R}, x_{C}+1\right) \\
& -k_{\mathrm{off}} x_{C} p_{t}\left(x_{L}, x_{R}, x_{C}\right) \\
& +k_{\mathrm{e}}\left(x_{C}+1\right) p_{t}\left(x_{L}, x_{R}, x_{C}+1\right) \\
& -k_{\mathrm{e}} x_{C} p_{t}\left(x_{L}, x_{R}, x_{C}\right) \\
& +k_{\mathrm{t}}\left(x_{R}+1\right) p_{t}\left(x_{L}, x_{R}+1, x_{C}\right) \\
& -k_{\mathrm{t}} x_{R} p_{t}\left(x_{L}, x_{R}, x_{C}\right) .
\end{aligned}
$$

\subsection{Sensitivity analysis of the model}

The proper measure of the modeled system is the reaction time in response to the change in the number of ligands. To enable comparison of the behavior of different receptors, the dimensionless relaxation time $\tau$ that indicates the rate of return to equilibrium after the introduction of a dose of ligands was defined as in Shankaran et al. (2007). Let $K_{D}=k_{\text {off }} / k_{\text {on }}$, using the following substitution:

$$
L^{*}=\frac{\langle \# L\rangle}{N_{A} V K_{D}}, \quad R^{*}=\frac{\langle \# R\rangle}{R_{T}}, \quad C^{*}=\frac{\langle \# C\rangle}{R_{T}},
$$

the deterministic version of the model was incorporated in order to transform the system of differential equations (37) into an equivalent form:

$$
\begin{aligned}
& \frac{\mathrm{d}}{\mathrm{d} t^{*}} L^{*}=\gamma\left(-R^{*} L^{*}+C^{*}\right)+f^{*} \\
& \frac{\mathrm{d}}{\mathrm{d} t^{*}} R^{*}=-R^{*} L^{*}+C^{*}+-\alpha\left(R^{*}-1\right) \\
& \frac{\mathrm{d}}{\mathrm{d} t^{*}} C^{*}=-R^{*} L^{*}-C^{*}+-\beta C^{*},
\end{aligned}
$$

where the dimensionless time was denoted by $t^{*}=t k_{\text {off }}$ and four new dimensionless parameters were introduced:

$$
\begin{array}{ll}
\alpha=\frac{k_{\mathrm{t}}}{k_{\mathrm{off}}} & \beta=\frac{k_{\mathrm{e}}}{k_{\mathrm{off}}} \\
\gamma=\frac{R_{T} k_{\mathrm{on}}}{k_{\mathrm{off}} N_{A} V} & f^{*}=\frac{f k_{\mathrm{on}}}{k_{\mathrm{off}}^{2}} .
\end{array}
$$

Let us consider the equilibrium state of a ligand-receptor model with no ligand molecules $L^{*}=0, R^{*}=1, C^{*}=0$ and suppose that at the initial time $t^{*}=0$ a single ligand dose was introduced into the system. The nature of the time evolution of a dimensionless average number of the cell surface complexes $C^{*}$ is the same as for the number of complexes of $C$ in the original model, i.e. 
the value of $C^{*}$ rapidly increases and after reaching the maximum value will slowly decline, seeking to balance state of the equilibrium. The point at which the value of $C^{*}$ falls below the value of maximum limit times $1 / e$ is called the dimensionless relaxation time and is denoted by $\tau$. A short relaxation time means a smooth operation of the system of cell membrane receptors, but the large values of $\tau$ indicate that the transmitted information (in case of receptors taking part in cellular signaling) or transmitted substance (for transporting receptors) will slowly reach the target cell. By conducting a formal SA of the receptors model, the relaxation time is taken as an outcome of the model and its sensitivity to the parameters disruption is examined.

To find the output value $\tau$ one must calculate the dimensionless coefficients according to formula (44). Assuming that the ligands inflow into the system appears only at the initial moment, we obtain $f^{*}=0$. The next computation step is the numerical solution of the equations system (43) with the initial conditions:

$$
L_{t^{*}=0}^{*}=0.01, \quad R_{t^{*}=0}^{*}=1, \quad C_{t^{*}=0}^{*}=0 .
$$

Let $C_{\max }^{*}$ denote the maximum value of $C^{*}$ reached at time point $t_{\max }^{*}$. Solving the interpolation problem $C^{*}(\tau)$ $=C_{\max }^{*} / e$ for $\tau>t_{\max }^{*}$, one receives a dimensionless relaxation time of the system.

As a matter of fact it is not necessary to solve the dimensionless system of differential equations (43), in order to calculate the output value $\tau$. It is enough to solve the system (37) (or equivalently (36)) with the initial conditions: $\langle \# L\rangle=0,01 K_{D} N_{A} V,\langle \# R\rangle=R_{\mathrm{T}},\langle \# C\rangle=0$, and then find the value $t>t_{\max }$ next to the time point $t_{\max }$ - the moment of the maximal complexes number $C_{\max }$, with $C(t)=C_{\max } / e$. After the scaling one obtains the dimensionless relaxation time $\tau=t k_{\text {off }}$.

In the same way one can find the value of $\tau$, using a stochastic version of the model. The relaxation time in this approach is a random variable. Using a stochastic simulation algorithm for the fixed parameters, one obtains the trajectory of a process of change of the number of substances in the system. Then, one finds a point $t$, in which the value of $C$ falls below 1/ $e$ of the maximum value. After multiplication of $t$ by $k_{\text {off }}$ one obtains the dimensionless relaxation time.

In the next sections of this paper, the local and the Morris method have been introduced for the classi- cal as well as the stochastic SA. For the deterministic version of the model, the global FAST methods were applied.

\subsubsection{Local methods}

We applied the local method of sensitivity analysis to the deterministic version of the ligand induced membrane receptors model for the previously mentioned four types of receptors. The aim of the analysis was to examine the influence of parameters $V, k_{\text {on }}, k_{\text {off }}, k_{\mathrm{e}}, k_{\mathrm{t}}$ and $R_{\mathrm{T}}$ on the dimensionless relaxation time $\tau$. The parameter $f$, denoting the rate of ligands production was omitted $(f=0)$, because a single dose of ligands entering the system was modeled by adopting the initial value $L^{*}$. The parameters values were set as in Table 1 and a change of parameters values $-10 \%$ increase was considered. The relaxation times for initial and disturbed values were numerically calculated from the equation (43) with the initial values (45).

Because the values of the measured parameters vary by several orders of magnitude, for the sensitivity measure the normalized local coefficients were used (15), multiplied by the face value of the parameter and divided by the nominal relaxation time. Results of our analysis are shown in Figure 2. Comparing the results obtained for different receptors, one can notice two common features of all studied systems. In each case, the parameter $k_{\mathrm{t}}$ turned out to be irrelevant to the relaxation time, as an outcome of the model. Furthermore, for each system, its sensitivity to the disturbance of parameter $k_{\text {on }}$ was the same as its sensitivity to the disturbance of parameter $R_{\mathrm{T}}$. Referring to the reactions scheme in the ligand induced receptor model (35) one can explain the first observed common feature. Both the production rate of membrane receptors and the rate of absorption of free receptors, are proportional to parameter $k_{\mathrm{t}}$. Since these are reverse reactions, the effect of parameter disturbance is equalized. The second observed regularity can easily be explained, referring the dimensionless form of the model (43) and noting that only the coefficient $\gamma$ depends on both parameters $k_{\text {on }}$ and $R_{\mathrm{T}}$ and is proportional to these parameters. Thus, sensitivity of the model to these parameters must be the same.

For the EGFR, LDLR and TfR, the same order of sensitivity to the parameters was obtained. The EGFR are highly local sensitive to disturbance of the parameter $k_{\text {off }}$ and moderately sensitive to disturbance of all other 

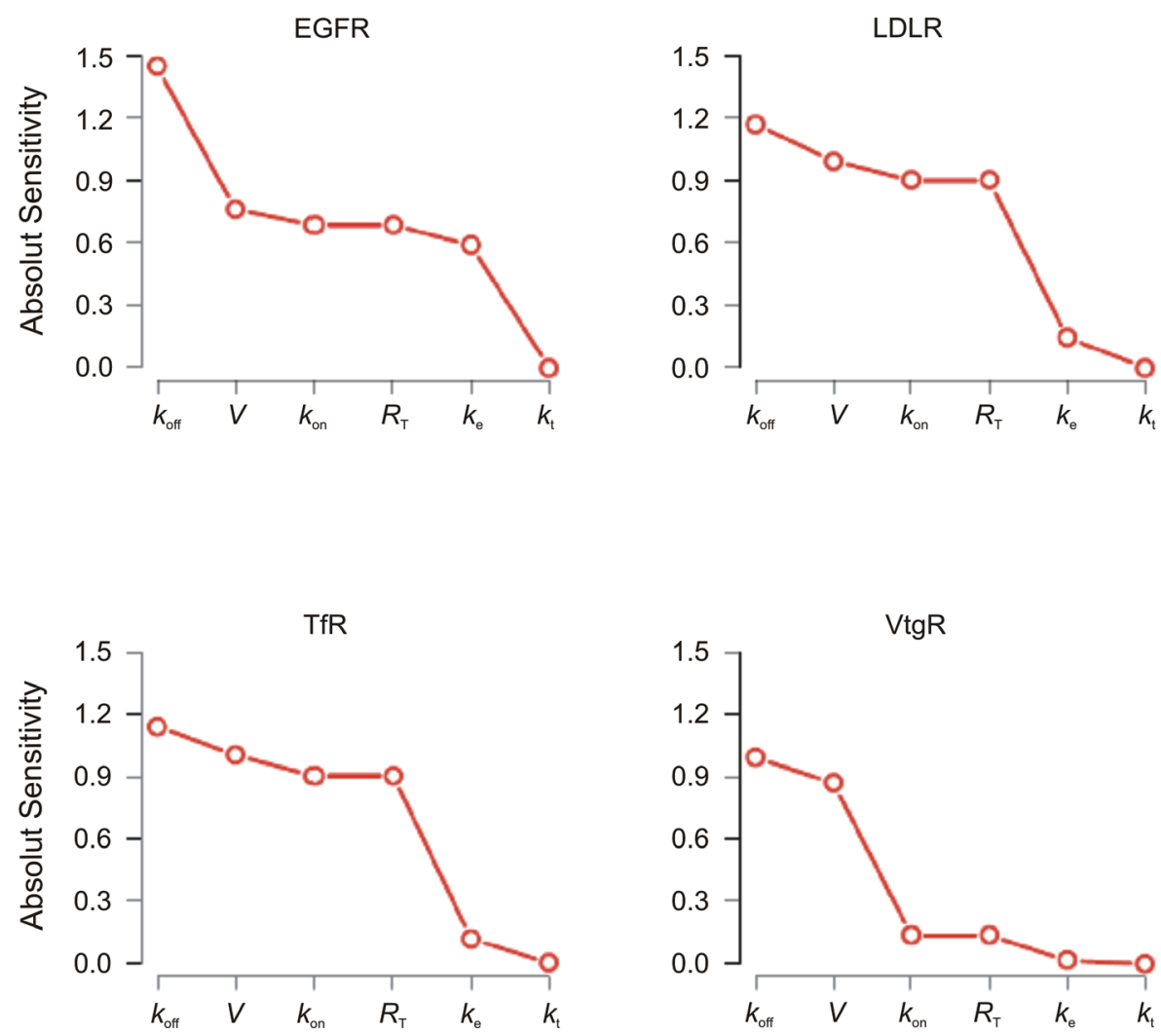

Fig. 2. Results of the local sensitivity analysis of the deterministic version of the ligand induced membrane receptors model. The vertical axis shows the normalized local sensitivity coefficients: $S_{i}=\tilde{x}_{i} / \tilde{\tau} \times \partial / \partial x_{i} \times \tau$, where $\tilde{x}_{i}$ denotes the face value of parameter $x_{i}$ and $\tilde{\tau}$ denotes the relaxation time for the face values parameters

parameters (except $k_{\mathrm{t}}$ ). Both transporting receptors LDLR and TfR showed similar sensitivity to parameter disturbances. Compared to other receptor systems, transport receptors LDLR and TfR exhibit low sensitivity to disturbance of the parameter $k_{\mathrm{e}}$. Different results were obtained for the receptor $\mathrm{VtgR}$ which proved to be most sensitive to disturbances of parameters $k_{\text {off }}$ and $k_{\mathrm{e}}$ and relatively low sensitive to changes in other parameter values. The results of the local SA correspond with the results presented in Shankaran et al. (2007), and with the results obtained for simulations carried out for the SA of the stochastic model of ligand induced membrane receptors.

Moreover, for the EGFR, we carried out a stochastic local SA. For this purpose, seven sets of parameters were considered - a set of face values of parameters, and six sets in which one of the six parameters were increased by $10 \%$. For each set, 5000 simulations of trajectories were performed, and on this basis dimensionless relaxation time was calculated according to the procedure described in the introduction of this section. For

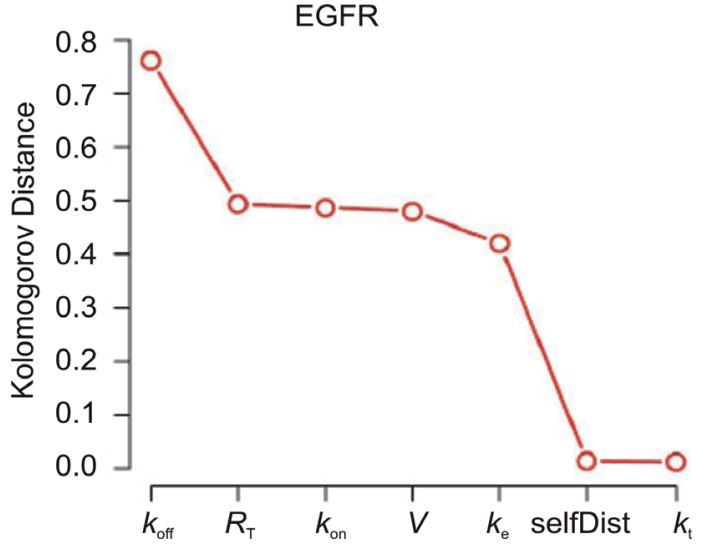

Fig. 3. Results of the local stochastic sensitivity analysis of the EGFR. On the vertical axis is the Kolmogorov distance between distributions of the relaxation time $\tau$ obtained for the nominal and increased by $10 \%$ parameters values. SelfDist means the distance of two samples generated for non-disturbed data.

Samples size was 5000

a set of face values, simulations were performed twice to determine the distance between histograms of two samples drawn from the same distribution. The result was 
5000 elements samples from distributions of the random variable $\tau$ for different parameters values. To compare these distributions, we used the Kolmogorov distance. The obtained results are illustrated in Figure 3.

The results of a local stochastic SA of the EGFR are the same as the results of the classical SA tailored for the deterministic version of the model. This confirms again that for the membrane receptors model, random fluctuations do not substantially affect the functioning of the system and that the deterministic model well describes its dynamics.

\subsubsection{Global method - FAST}

Using the FAST algorithm, we examined the sensitivity of the absolute relaxation time on the disturbance of parameters: $V, k_{\text {on }}, k_{\text {off }}, k_{\mathrm{e}}$ and $R_{\mathrm{T}}$. The results of the analysis are shown in Table 2 and in Figures 4 and 5. The first column of figures contains the estimators of sensitivity coefficients $\hat{S}_{i}$ for the studied systems of membrane receptors. The second column illustrates the differences between the obtained total sensitivity coefficients and firstorder sensitivity coefficients $\hat{S}_{i}^{T}-\hat{S}_{i}$.

The analysis of the results of the FAST method leads to two important conclusions common for all the receptor systems. First of all, for each tested receptor, the sum of sensitivity coefficients $S_{i}$ is close to unity. Thus, the differences between the total sensitivity coefficients and the first-order sensitivity coefficients are close to zero. This means that in the studied systems, the impact of the interaction between the parameters on the dimensionless relaxation time is negligibly small and the model behaves in a linear fashion.

Furthermore, for each tested receptor, parameter $k_{\text {off }}$ was the most important for the relaxation time.

In the EGFR system, parameter $k_{\text {off }}$ explains almost $50 \%$ of the total variance of the model, while the remainder of the variance is more or less equally distributed between the other parameters. Both transporting recep- tors, LDLR and TfR, have the same pattern of sensitivity, which indicates a strong connection between the dynamics of modeled reactions performed by receptors and their role in functioning of the organism. A characteristic feature of these systems is non-dependence of the relaxation time on the parameter $k_{\mathrm{e}}$. The influence of other parameters on the variance of the model outcome is moderate and ranges from 20 to $30 \%$. However, in the case of receptor VtgR, the only parameters that affect the relaxation time of the system, are $k_{\text {off }}$ and $k_{\mathrm{e}}$. Their combined effect on the outcome of the model exceeds $97 \%$ of the total variance.

It should be remembered that the coefficient $\beta$ is defined as the ratio of parameters $k_{\mathrm{e}}$ and $k_{\text {off }}(44)$. The parameter $k_{\mathrm{e}}$ denotes the rate of endocytosis of ligandreceptor complexes, whereas the value $k_{\text {off }}$ is the rate of disintegration of the complex to free ligand and free receptor. Thus, the coefficient $\beta$ can be interpreted as the probability that the bound ligand is absorbed into the cell before it detaches from the receptor. The value $\gamma$ is proportional to $k_{\text {on }}$ the rate of binding into complexes and to the number of free receptors on the membrane denoted by the parameter $R_{\mathrm{T}}$ and inversely proportional to $k_{\text {off }}$ the rate of disintegration of the complexes and volume of the system $V$. The value of coefficient $\gamma$ indicates how well a receptor can catch free ligands from the intercellular space. Therefore, dimensionless coefficients $\beta$ and $\gamma$ characterize two independent and complementary features of membrane receptors, i.e. the ability to capture ligands from the intercellular space and the effectiveness of their absorption into the cell. The results of the SA of the model outcome to the dimensionless parameters are shown in Table 3 and Figure 6.

Also in the case of an SA of the dimensionless version of the membrane receptors model, the sum of the first-order sensitivity coefficients is close to unity, so the interaction between the dimensionless parameters

Table 2. Results of the global sensitivity analysis of the receptor model using the FAST algorithm

\begin{tabular}{c|c|c|c|c|c|c|c|c}
\hline & \multicolumn{2}{|c|}{ EGFR } & \multicolumn{2}{c|}{ LDLR } & \multicolumn{2}{c}{ TfR } & \multicolumn{2}{c}{ VtgR } \\
\hline & $S_{i}$ & $S_{i}^{T}$ & $S_{i}$ & $S_{i}^{T}$ & $S_{i}$ & $S_{i}^{T}$ & $S_{i}$ & $S_{i}^{T}$ \\
\hline$V$ & 0.143 & 0.146 & 0.215 & 0.219 & 0.218 & 0.222 & 0.003 & 0.008 \\
\hline$k_{\text {on }}$ & 0.144 & 0.148 & 0.238 & 0.241 & 0.241 & 0.245 & 0.003 & 0.008 \\
\hline$k_{\text {off }}$ & 0.483 & 0.487 & 0.309 & 0.314 & 0.300 & 0.305 & 0.528 & 0.533 \\
\hline$k_{\mathrm{e}}$ & 0.093 & 0.096 & 0.007 & 0.008 & 0.005 & 0.006 & 0.449 & 0.456 \\
\hline$R_{\mathrm{T}}$ & 0.138 & 0.141 & 0.215 & 0.219 & 0.218 & 0.222 & 0.002 & 0.008 \\
\hline
\end{tabular}



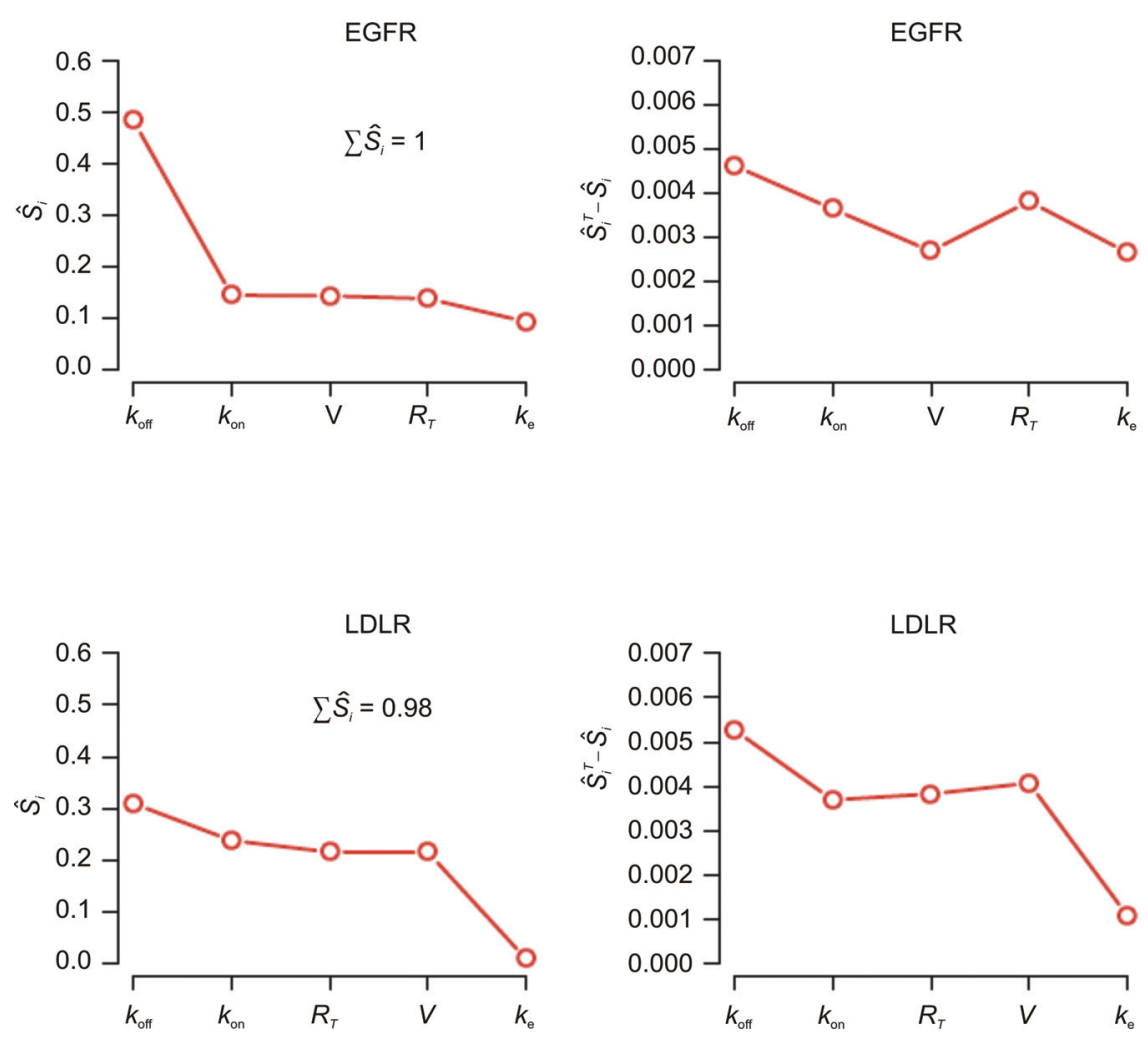

Fig. 4. Results of the global sensitivity analysis of the EGFR and LDLR receptor models using the FAST algorithm
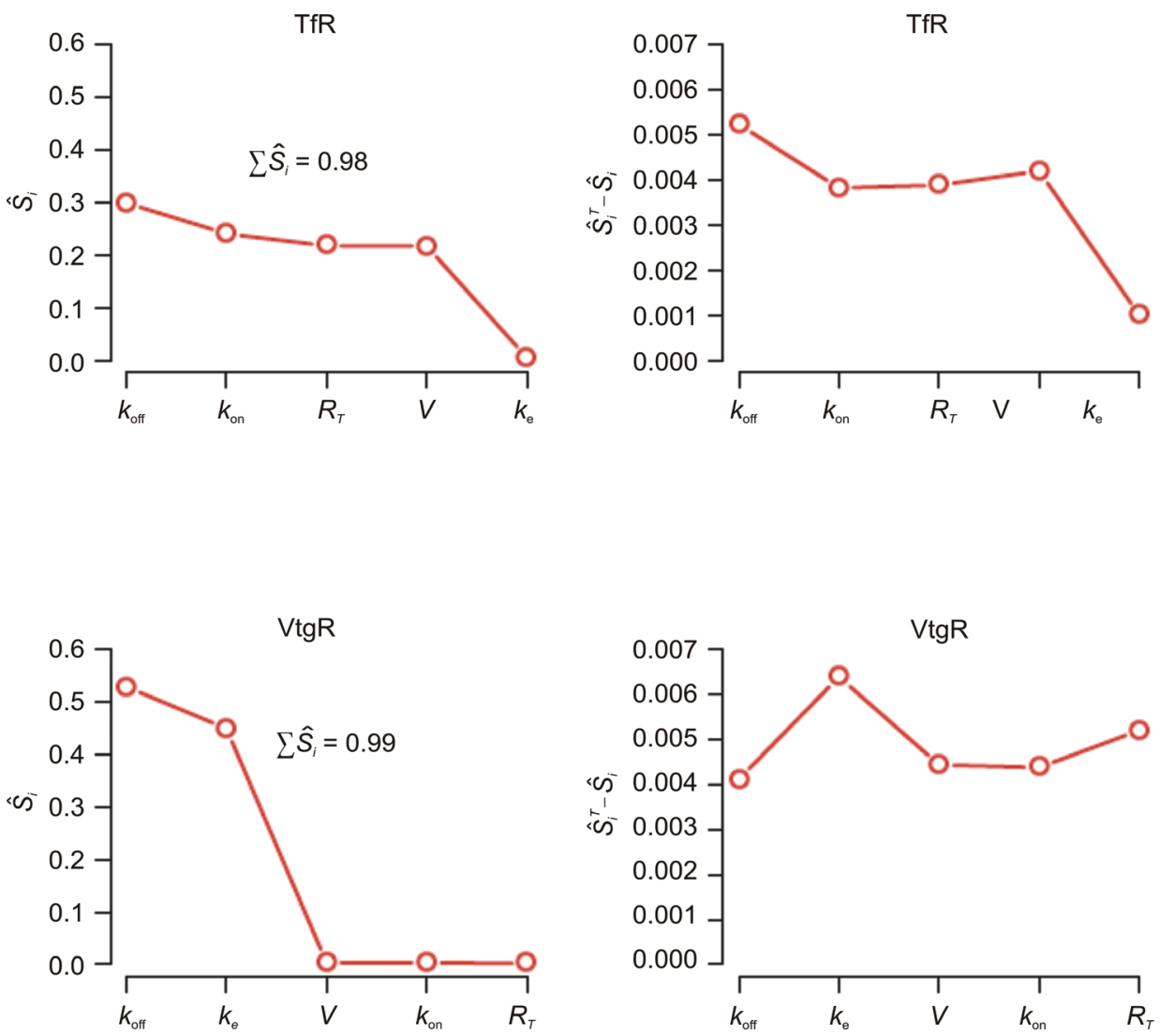

Fig. 5. Results of the global sensitivity analysis of the TfR and VtgR receptor models using the FAST algorithm 
Table 3. Results of the global sensitivity analysis of the membrane receptors model conducted using an extended version of the FAST algorithm. The dimensionless relaxation time $\tau$ as a function of dimensionless coefficients $\alpha, \beta$ and $\gamma$

\begin{tabular}{c|c|c|c|c|c|c|c|c}
\hline & \multicolumn{2}{|c|}{ EGFR } & \multicolumn{2}{c|}{ LDLR } & \multicolumn{2}{c}{ TfR } & \multicolumn{3}{c}{ VtgR } \\
\hline & $S_{i}$ & $S_{i}^{T}$ & $S_{i}$ & $S_{i}^{T}$ & $S_{i}$ & $S_{i}^{T}$ & $S_{i}$ & $S_{i}^{T}$ \\
\hline$\alpha$ & 0.000 & 0.001 & 0.000 & 0.002 & 0.000 & 0.002 & 0.000 & 0.000 \\
\hline$\beta$ & 0.416 & 0.419 & 0.024 & 0.027 & 0.017 & 0.019 & 0.985 & 0.991 \\
\hline$\gamma$ & 0.582 & 0.585 & 0.972 & 0.975 & 0.980 & 0.983 & 0.010 & 0.017 \\
\hline
\end{tabular}
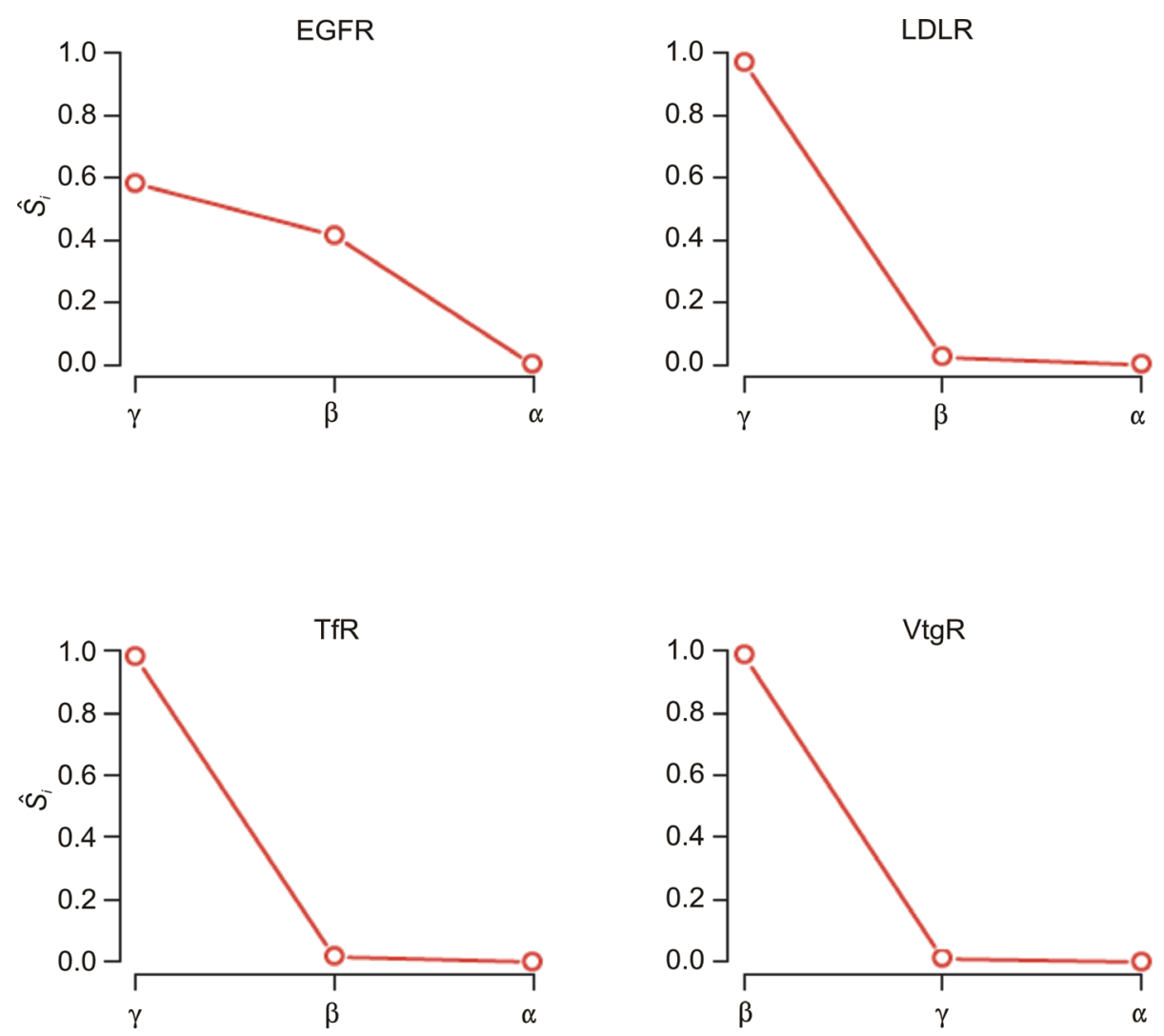

Fig. 6. Results of the global sensitivity analysis of the membrane receptors model conducted using the FAST algorithm.

The dimensionless relaxation time $\tau$ as a function of dimensionless coefficients $\alpha, \beta$ and $\gamma$

has no significant impact on the relaxation time. In each of the studied systems, the importance of the factor $\alpha$ is negligibly small. This regularity results from the definition of coefficient $\alpha$ equal to the quotient of the parameters $k_{\mathrm{t}}$ and $k_{\text {off }}$ and lack of materiality of parameter $k_{\mathrm{t}}$ shown by the local SA.

The obtained results show different ways of functioning of various systems of membrane receptors. For the EGFR, which is involved in cell signaling, equally important is the ability to capture ligands from the intercellular space, as well as the ability to effectively communicate the information to the target cell. The proper functioning of transporting receptors LDLR and TfR depends entirely on the effective catching of ligands. However, in the case of VtgR, the only important feature appeared to be the ability to carry out the process of endocytosis. The dimensionless form of the SA of a membrane receptors model enabled better understanding of the operational principles of the considered systems.

\subsubsection{Screening method - Morris method}

Finally, we applied the Morris method in the SA. The aim was to investigate the dependence of the dimensionless relaxation time $\tau$ on parameters $V, k_{\text {on }}, k_{\text {off }}$, $k_{\mathrm{e}}$ and $R_{\mathrm{T}}$. As before, the parameters $f$ and $k_{\mathrm{t}}$ were omit 

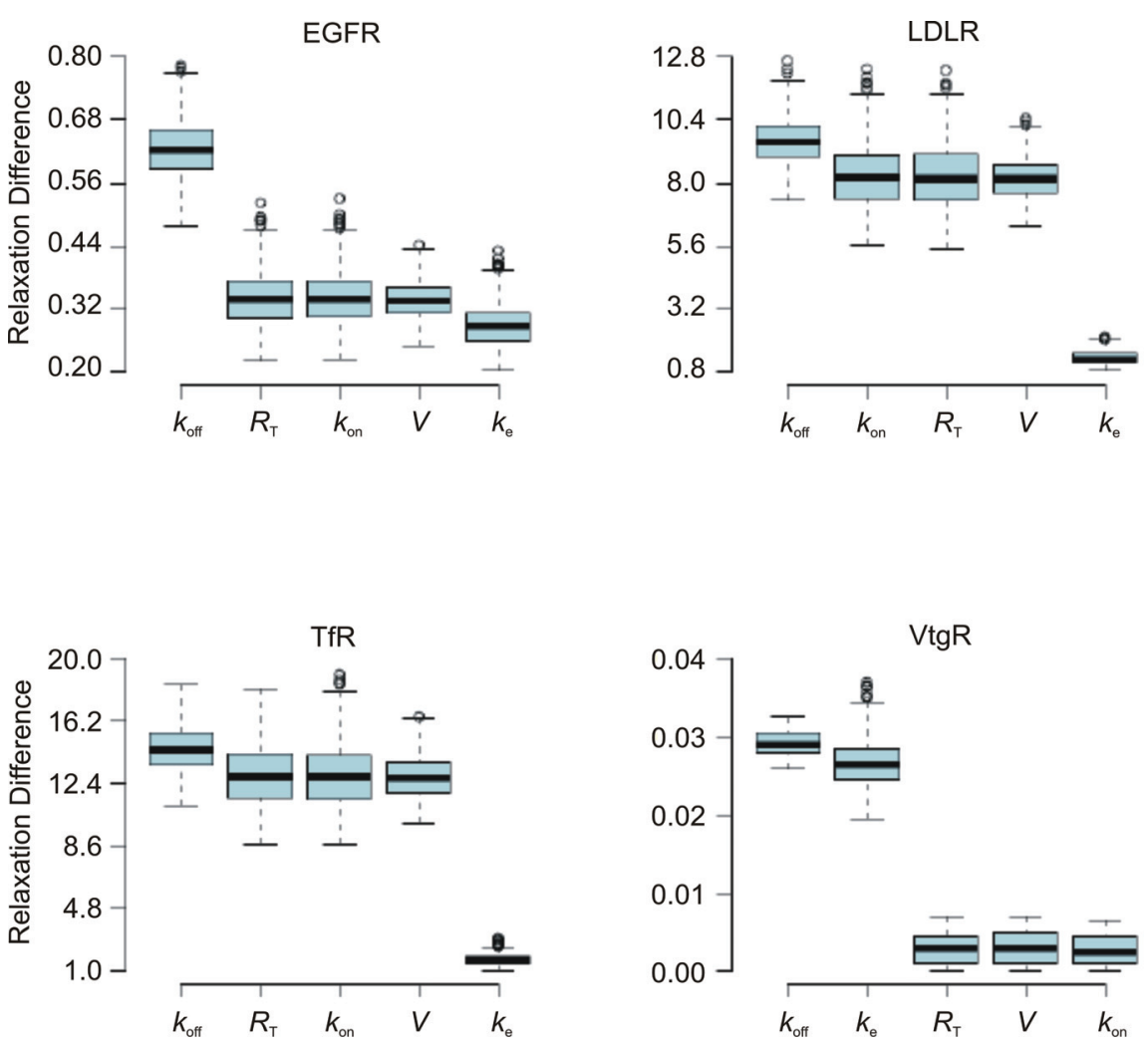

Fig. 7. Results of the Morris method for the deterministic version of the model of membrane receptors. The parameter values were drawn from the set $\left\{90 \% \tilde{X}_{i}, 92 \% \tilde{X}_{i}, \ldots, 110 \% \widetilde{X}_{i}\right\}$, where $\widetilde{X}_{i}$ denotes the face value. For each parameter, the sample size was 1000

ted, because the local SA showed that their effect on the outcome of the model is irrelevant.

The range of variation of each parameter was set at $90 \%$ to $110 \%$ of its face value $\tilde{x}_{i}$. We assumed that the parameter can obtain with equal probability one of the following values: $\left\{90 \% \tilde{X}_{i}, 92 \% \tilde{X}_{i}, \ldots, 110 \% \tilde{X}_{i}\right\}$. Consequently, we obtain a 5-dimensional, 11-leveled network $\omega$ of parameter values.

For each parameter, the set of 1000 mesh points was drawn independently from $\omega$ excluding the edges points. We solved the system of dimensionless equations (43) with initial conditions (45), finding the value of $\tau$ for the sets of parameters corresponding to the points in the grid one notch to the left and the right of each drawn point and calculating the differences in relaxation times. In Figure 7, the average value and dispersion of the absolute difference in relaxation times was shown.

The obtained parameter order, arranged according to their decreasing effect on the outcome of the model, is different from that obtained in the local SA. More important, however, is the fact, that once the results are classified into parameter groups of significant, modera- tely significant and irrelevant for the relaxation time, the results of both methods are consistent.

We also conducted a stochastic SA of the EGFR model with the Morris method. The results are presented in Figure 8 . The order of parameters for the EGFR model indicated by the stochastic version of the Morris algorithm is different than that achieved by the classical method, but the classification of parameters to significant,

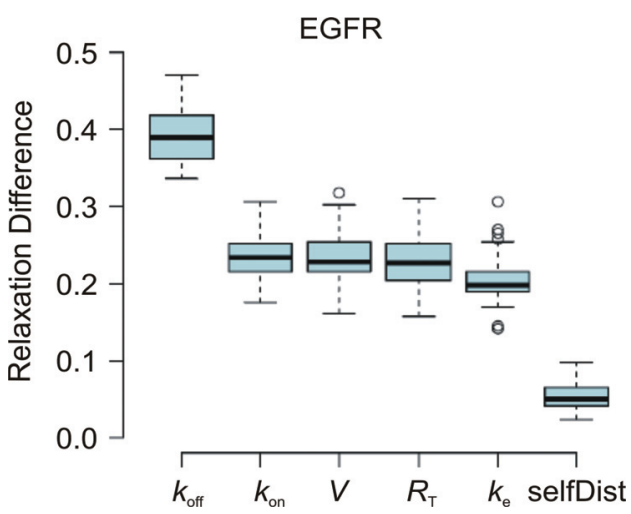

Fig. 8. Results of the stochastic sensitivity analysis of the EGFR model conducted with Morris method. SelfDist means the distance of two samples generated for non-disturbed data 
moderately significant and insignificant has been preserved. Thus, also in this case, the deterministic model well enough describes the processes occurring in the system of membrane receptors.

\section{Conclusions}

Transport of particles into the cell as well as cell signaling is carried out by receptors located on the cell membrane. This paper focuses on mathematical modeling of interactions in the system of membrane receptors. We have presented the modeling framework using the exemplary model by Shankaran et al. (2007). To illustrate the stochastic approach, we transformed the system of differential equations describing the dynamics of the system into a continuous time Markov chain governed by a chemical master equation. We conducted the SA for both models using various methods reviewed in the paper. The analysis concerned the relaxation time (i.e. the time taken for the impulse response to decay to a value of $1 / \mathrm{e}$ of the peak value). This parameter well characterizes the receptor system performance: e.g. a lower relaxation time implies a faster response to the presence of ligands. Four kinds of experimentally investigated receptors were considered, i.e. epidermal growth factor receptor, transferrin receptor, low-density lipoprotein receptor and vitellogenin receptor.

In all cases, the conclusions based on the results of SA justified the use of a deterministic framework. Moreover, some of the parameters were identified as having a negligible impact on the system performance. Last, but not least, the SA of the membrane receptors model provided an insight into the operational principles of the studied systems.

\section{Acknowledgments}

This work was partially supported by the Polish National Science Center grant 2011/01/B/NZ2/00864 and by the Biocentrum Ochota project POIG.02.03.00-00-003/09. MR is a scholar within the Human Capital Operational Programme co-financed by European Social Fund and state budget. The paper is cofunded by the European Union from resources of the European Social Fund. Project PO KL Information technologies: Research and their interdisciplinary applications.
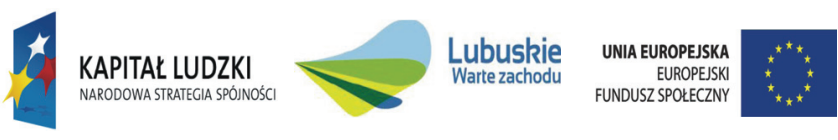

\section{References}

Bentele M., Lavrik I., Ulrich M., Stösser S., Heermann D.W., Kalthoff H., Krammer P.H., Eils R. (2004) Mathematical modeling reveals threshold mechanism in CD95-induced apoptosis. J. Cell Biol. 166: 839-851.

Butcher J.C. (2003) Numerical Methods for Ordinary Differential Equations. Wiley.

Campolongo F., Saltelli A., Tarantola S. (2000) Sensitivity analysis as an ingredient of modeling. Statistic. Sci. 15: 377-395.

Cukier R.I., Levine H.B., Shuler K.E. (1978) Nonlinear sensitivity analysis of multiparameter model systems. J. Comput. Phys. 26: 1-42.

Degasperi A., Gilmore S. (2008) Sensitivity analysis of stochastic models of bistable biochemical reactions. Lect. Notes Comput. Sci. 5016: 1-20.

Gillespie D.T. (1992) A rigorous derivation of the chemical master equation. Physica A: Stat. Mechan. Applic. 188: 404-425.

Gillespie D.T. (2000) The chemical Langevin equation. J. Chem. Phys. 113: 297-306.

Goutsias J. (2007) Classical versus stochastic kinetics modeling of biochemical reaction systems. Biophys. J. 92: 2350-2365.

Gunawan R., Cao Y., Petzold L., Doyle F.J. (2005) Sensitivity analysis of discrete stochastic systems. Biophys. J. 88: 2530-2540.

Hornberger G.M., Spear R.C. (1981) An approach to the preliminary analysis of environmental systems. J. Environ. Manag. 12: 7-18.

Kampen N.V. (2007) Stochastic Processes in Physics and Chemistry, Third Edition. North Holland.

Mahdavi A., Davey R.E., Bhola P., Yin T., Zandstra P.W. (2007) Sensitivity analysis of intracellular signaling pathway kinetics predicts targets for stem cell fate control. PLoS Comput. Biol. 3: e130.

Morris M.D. (1991) Factorial sampling plans for preliminary computational experiments. Technometr. 33: 161-174.

Saltelli A., Ratto M., Andres T., Campolongo F., Cariboni J., Gatelli D., Saisana M., Tarantola S. (2008) Global Sensitivity Analysis: The Primer. Wiley-Interscience.

Saltelli A., Ratto M., Tarantola S., Campolongo F. (2005) Sensitivity analysis for chemical models. Chem. Rev. 105: 2811-2828.

Saltelli A., Tarantola S., Chan K.P.S. (1999) A quantitative model-independent method for global sensitivity analysis of model output. Technometrics 41: 39-56.

Shankaran H., Resat H., Wiley H.S. (2007) Cell surface receptors for signal transduction and ligand transport: a design principles study. PLoS Comput. Biol. 3: 0986-0999.

Shankaran H., Wiley H.S., Resat H. (2006) Modeling the effects of HER/ErbB1-3 coexpression on receptor dimerization and biological response. Biophys. J. 90: 3993-4009.

Sjöberg P., Lötstedt P., Elf J. (2009) Fokker-Planck approximation of the master equation in molecular biology. Comput. Visual. Sci. 12: 37-50. 
Sobol I.M. (1993) Sensitivity analysis for nonlinear mathematical models. Mathem. Model. Comput. Exper. 1: 407-414.

Sobol I.M., Kucherenko S. (2005) Global sensitivity indices for nonlinear mathematical models. Review. Wilmott 1: 2-7.

Turanyi T. (1990) Sensitivity analysis of complex kinetic systems. tools and applications. J. Mathem. Chem. 5: 203-248.
Wolkenhauer O., Ullah M., Kolch W., Cho K.H. (2004) Modeling and simulation of intracellular dynamics: Choosing an appropriate framework. IEEE Trans. Nanobiosci. 3: 200-207.

Yue H., Brown M., He F., Jia J., Kell D. (2008) Sensitivity analysis and robust experimental design of a signal transduction pathway system. Intern. J. Chem. Kinet. 40: 730-741. 